\title{
A systematic nomenclature for mammalian tropomyosin isoforms
}

\author{
Michael A. Geeves • Sarah E. Hitchcock-DeGregori • \\ Peter W. Gunning
}

Received: 22 May 2014/ Accepted: 8 September 2014/Published online: 5 November 2014

(c) The Author(s) 2014. This article is published with open access at Springerlink.com

\begin{abstract}
Tropomyosin, a ubiquitous protein in animals and fungi, is associated with the actin cytoskeleton and is involved with stabilising actin filaments and regulating the interaction of the filament with other actin binding proteins. The protein is best known for its role in regulating the interaction between actin and myosin in muscle contraction but in recent years its role as a major player in the organisation and dynamics of the cytoskeleton has been increasingly recognised. In mammals Tpm is expressed from four distinct genes and alternate splicing of each gene can produce a total of up to 40 different mRNA variants most of which are expressed as proteins. We are expecting a renaissance in the study of tropomyosins as the roles of these different isoforms are beginning to be deciphered. However, it is our belief that such a renaissance is being limited by confusion over the naming systems for the tropomyosin isoforms. These result in even experienced workers struggling to reconcile work done in different laboratories and at different times. We propose here a systematic nomenclature for tropomyosin based on the best current practice. We recommend the adoption of these
\end{abstract}

All authors contributed equally to this work

M. A. Geeves $(\square)$

School of Biosciences, University of Kent, Canterbury CT2 7NJ, UK

e-mail: m.a.geeves@ukc.ac.uk; m.a.geeves@kent.ac.uk

S. E. Hitchcock-DeGregori

Robert Wood Johnson Medical School, Rutgers University, Piscataway, NJ 08854, USA

P. W. Gunning

School of Medical Sciences, University of New South Wales (UNSW), Sydney, NSW 2052, Australia names and a cross-reference to the table of alternate names and accession numbers for protein sequences is included here. The National Center for Biotechnology Information (NCBI) website has been amended to include the nomenclature for the human, mouse and rat genes.

Keywords Cytoskeleton - Actin binding protein $\cdot$ Muscle thin filament

Tropomyosin (Tpm) is a protein family associated with the stabilisation and regulation of the actin cytoskeleton (Gunning et al. 2008). It is expressed in opisthokonts including animals and fungi, but is not documented in plants, protists or procaryotes (Barua et al. 2011; CranzMileva et al. 2013). Tpm polymerises head to tail as a twochained coiled coil along both sides of a helical actin filament stabilising the actin structure and interacting with a range of other actin binding proteins to regulate the actin cytoskeleton. The protein was discovered in the 1940s as a component of the actin filaments of striated muscle (Bailey 1946) and this remains the best studied example of Tpm function. In striated muscle, together with troponin, it enables calcium regulation of striated muscle contraction by blocking $\left(-\mathrm{Ca}^{2+}\right)$ or allowing $\left(+\mathrm{Ca}^{2+}\right)$ myosin access to its binding site on actin (Gordon et al. 2000; Geeves 2012). Since the 1940s the different possible roles of Tpm and the number of interacting partners has multiplied (see special issue of $\mathrm{J}$ Muscle Res. Cell Motil., Marston \& Gautel 2013). We now know that in mammalian cells Tpm is expressed from four separate genes and alternate promoter selection and splicing of each gene can produce up to 40 different mRNA variants most of which have been shown to be expressed as proteins in different tissues (Pittenger et al. 1994; Vindin and Gunning 2013). 
The steady discovery of different splice variants in specific tissues has, over the years, led to a proliferation of different names for the isoforms, some identified with specific tissues but later found with a broader distribution. This has resulted in a great deal of confusion in the literature and it is often difficult, even for those of us in the field, to be sure which isoforms we are each referring to. It must be an even bigger nightmare for those trying to enter the field. The lack of a coherent nomenclature is an impediment to progress. We firmly believe that Tpm research is due for a renaissance from both the structurefunction perspective in muscle research and the cytoplasmic perspective in the localisation-function of distinct isoforms and their binding partners. This is evidenced by the collection of articles published in a special issue of $\mathbf{J}$ Muscle Res Cell Motil devoted to Tpm Marston \& Gautel (2013). This renaissance will be enhanced if we can establish a widely agreed-upon nomenclature that the majority of active researchers will use. It will be much better if we can establish this now as the field begins to expand and other pressures develop on the nomenclature.

After consulting colleagues working with Tpm we now make a specific proposal for how the nomenclature could be simplified and rationalised and to make it compatible with current best practice for gene and protein names. The proposal keeps as close as possible to some well-established historical precedents.

Our suggestion is that any publication dealing with the tropomyosin protein should designate, at the first use, the formal name of the protein isoform as proposed here, and then define the short form or abbreviation that will be used thereafter in the article text. Reference to Tables 1, 2, 3 and 4 here will allow cross-reference to any earlier versions of the names used e.g., $\alpha$-fast skeletal tropomyosin is Tpm1.1.

\section{Detailed proposals}

1. The human tropomyosin genes should be known as TPM1 through TPM4 (Tpm1 through Tpm4 for mouse and rat tropomyosin) to be consistent with other gene nomenclatures.

2. The protein short name Tm is historically well established but Tpm is consistent with standard protein nomenclature and is therefore preferred in the formal name.

3. Tpm1 or Tpm2 specifies the protein is from gene TPM1 or TPM2 etc.

4. Alternate isoforms are numbered systematically from one (e.g., Tpm1.1 etc.), keeping as close as possible to any precedent. Thus Tpm2.3 is the tropomyosin isoform from gene 2 isoform 3 .

5. In the formal name a subscript designates the tissue most closely associated, historically, with the protein isoform. Many such isoforms have been since found in multiple tissues and such designations are not always useful.

- St striated muscle, cardiac/skeletal tissue

- Sm smooth muscle,

- Br brain

- Cy other cytoplasmic

6. The splicing of the four exons that vary in vertebrates can be designated by a four letter code; a.a.b.d indicates exon 1 is splice form a, exon 2 is splice form a, exon 6 is splice form $b$ and exon 9 is splice form $\mathrm{d}$.

- A dash as in b.-.b.d indicates that exon 2 is missing and therefore this is a short form of Tpm.

- Previous publications of Tpm isoforms have indicated that exon 9 can be a combination of two splice forms at the mRNA level (e.g., Vindin and Gunning 2013) but these reflect splice variants in non-coding regions at the $3^{\prime}$ end of the mRNA transcripts. We have therefore omitted such isoforms from our list of expressed proteins.

7. The formal names of the two tropomyosins from the TPM1 gene, found in the contractile filaments of smooth muscle, are then

$$
\mathrm{Tpm} 1.3_{\mathrm{Sm} \text { (a.a.b.d) }} \text { and } \mathrm{Tpm} 1.4_{\mathrm{Sm} \text { (a.a.a.d) }}
$$

The short form of the name would be Tpm1.3 and Tpm1.4

Tables 1, 2, 3 and 4 list the current, known Tpm protein isoforms and the proposed formal names. As far as possible the alternate names for the same protein that have been used in the past are included in columns two and three. We also include, in Table 5, the full set of human Tpm amino acid sequences in the order that the exons appear in the gene. This will allow any ambiguity in the literature to be checked against the names and exon sequences used here. A fuller list of animal Tpm sequences can be found in Barua et al. 2011 and Schevzov et al. 2011.

It is almost certain that additional isoforms of tropomyosin will be identified in these species. Consideration of all possible splicing combinations already established in at least one isoform leads to a calculation of 48 possible distinct isoforms in humans without consideration of currently unknown splicing alternatives. For example, Cooley and Bergtrom (2001) identified an increased number of 
Table 1 List of tropomyosin isoforms derived from the human TPM1 gene (geneID:7168), the mouse Tpm1 gene(geneID:22003) or the rat Tpm 1 gene (geneID:24851). Gene structure

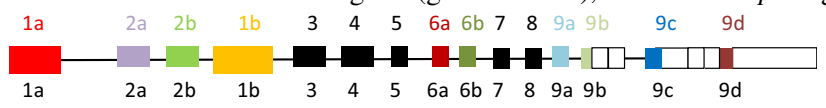

\begin{tabular}{|c|c|c|c|c|c|}
\hline $\begin{array}{l}\text { Short } \\
\text { name }\end{array}$ & $\begin{array}{l}\text { Common } \\
\text { names }\end{array}$ & Alternate names in use & $\begin{array}{l}\text { Formal protein } \\
\text { name }\end{array}$ & $\begin{array}{l}\text { Accession numbers } \\
\text { Full length sequences only }\end{array}$ & Exon usage $^{\mathrm{b}}$ \\
\hline Tpm1.1 & Tm sk $\alpha$ & $\begin{array}{l}\alpha \mathrm{Tm}, \text { fast } \mathrm{skTm}, \\
\quad \text { cardiac } \mathrm{Tm}, \text { striated } \mathrm{Tm}\end{array}$ & Tpm1.1st (a.b.b.a) & $\begin{array}{l}\text { Human: NP_001018005.1 } \\
\text { Mouse: NP_001157720.1 } \\
\text { Rat: NP_001288265.1 }\end{array}$ & $\begin{array}{l}1 \mathrm{a} \cdot 2 \mathrm{~b} \cdot 3 \cdot 4 \cdot 5 \cdot 6 \mathrm{~b} \cdot 7 \cdot 8 \cdot 9 \mathrm{a} \\
1 \mathrm{a} \cdot 2 \mathrm{~b} \cdot 3 \cdot 4 \cdot 5 \cdot 6 \mathrm{~b} \cdot \mathbf{7} \cdot 8 \cdot 9 \mathrm{a}\end{array}$ \\
\hline Tpm1.2 & Tm sk $\alpha 1$ & $\kappa \mathrm{Tm}$, Tmsk $\alpha 1-1$ & Tpm1.2st (a.a.b.a) & $\begin{array}{l}\text { Human: } \\
\text { NP_001288173.1 } \\
\text { Mouse: none } \\
\text { Rat: NP_001288271.1 }\end{array}$ & $\begin{array}{l}1 a \cdot 2 a \cdot 3 \cdot 4 \cdot 5 \cdot 6 b \cdot 7 \cdot 8 \cdot 9 a \\
1 a \cdot 2 a \cdot 3 \cdot 4 \cdot 5 \cdot 6 b \cdot 7 \cdot 8 \cdot 9 a\end{array}$ \\
\hline Tpm1.3 & $\operatorname{Tm} \operatorname{sm} \alpha-1$ & $\alpha$ smooth Tm & Tpm1.3sm (a.a.a.d) & $\begin{array}{l}\text { Human: NP_001018020.1 } \\
\text { Mouse: none } \\
\text { Rat: NP_001029243.1 }\end{array}$ & $\begin{array}{l}1 a \cdot 2 a \cdot 3 \cdot 4 \cdot 5 \cdot 6 a \cdot 7 \cdot 8 \cdot 9 d \\
1 a \cdot 2 a \cdot 3 \cdot 4 \cdot 5 \cdot 6 a \cdot 7 \cdot 8 \cdot 9 d\end{array}$ \\
\hline Tpm1.4 & $\operatorname{Tm} \operatorname{sm} \alpha$ & $\begin{array}{l}\text { Tm6, } \alpha \text { smooth Tm } \\
\operatorname{Tm} \alpha 1.2\end{array}$ & Tpm1.4sm (a.a.b.d) & $\begin{array}{l}\text { Human: NP_001018007.1 } \\
\text { Mouse: NP_001157721.1 } \\
\text { Rat: AAA21804.1 }\end{array}$ & $\begin{array}{l}1 \mathrm{a} \cdot 2 \mathrm{a} \cdot 3 \cdot 4 \cdot 5 \cdot 6 \mathrm{~b} \cdot 7 \cdot 8 \cdot 9 \mathrm{~d} \\
1 \mathrm{a} \cdot 2 \mathrm{a} \cdot 3 \cdot 4 \cdot 5 \cdot 6 \mathrm{~b} \cdot 7 \cdot 8 \cdot 9 \mathrm{~d}\end{array}$ \\
\hline Tpm1.5 & $\operatorname{Tm} 3-1$ & - & Tpm1.5cy (a.b.a.a) & $\begin{array}{l}\text { Human: NP_000357.3 } \\
\text { Mouse: NP_001157723.1 } \\
\text { Rat: none }\end{array}$ & $\begin{array}{l}1 \mathrm{a} \cdot 2 \mathrm{~b} \cdot 3 \cdot 4 \cdot 5 \cdot 6 \mathrm{a} \cdot 7 \cdot 8 \cdot 9 \mathrm{a} \\
1 \mathrm{a} \cdot 2 \mathrm{~b} \cdot 3 \cdot 4 \cdot 5 \cdot 6 \mathrm{a} \cdot 7 \cdot 8 \cdot 9 \mathrm{a}\end{array}$ \\
\hline Tpm1.6 & $\operatorname{Tm} 2$ & - & Tpm1.6cy (a.b.b.d) & $\begin{array}{l}\text { Human: NP_001018004.1 } \\
\text { Mouse: NP_077745.2 } \\
\text { Rat: NP_001029241.1 }\end{array}$ & $\begin{array}{l}1 \mathrm{a} \cdot 2 \mathrm{~b} \cdot 3 \cdot 4 \cdot 5 \cdot 6 \mathrm{~b} \cdot 7 \cdot 8 \cdot 9 \mathrm{~d} \\
1 \mathrm{a} \cdot 2 \mathrm{~b} \cdot 3 \cdot 4 \cdot 5 \cdot 6 \mathrm{~b} \cdot 7 \cdot 8 \cdot 9 \mathrm{~d}\end{array}$ \\
\hline Tpm1.7 & $\operatorname{Tm} 3$ & $\operatorname{Tm} 1.4$ & Tpm1.7cy (a.b.a.d) & $\begin{array}{l}\text { Human: NP_001018006.1 } \\
\text { Mouse: NP_001157722.1 } \\
\text { Rat: NP_001029242.1 }\end{array}$ & $\begin{array}{l}1 \mathrm{a} \cdot 2 \mathrm{~b} \cdot 3 \cdot 4 \cdot 5 \cdot 6 \mathrm{a} \cdot 7 \cdot 8 \cdot 9 \mathrm{~d} \\
1 \mathrm{a} \cdot 2 \mathrm{~b} \cdot 3 \cdot 4 \cdot 5 \cdot 6 \mathrm{a} \cdot 7 \cdot 8 \cdot 9 \mathrm{~d}\end{array}$ \\
\hline Tpm1.8 & $\operatorname{Tm} 5 \mathrm{a}$ & - & Tpm1.8cy (b.-.b.d) & $\begin{array}{l}\text { Human: NP_001288218.1 } \\
\text { Mouse: NP_001157724.1 } \\
\text { Rat: NP_001029245.1 }\end{array}$ & $\begin{array}{l}1 \mathrm{~b} .-3.4 .5 .6 \mathrm{~b} .7 .8 .9 \mathrm{~d} \\
1 \mathrm{~b} .-3.4 .5 .6 \mathrm{~b} .7 .8 .9 \mathrm{~d}\end{array}$ \\
\hline Tpm1.9 & $\operatorname{Tm} 5 b$ & - & Tpm1.9cy (b.-.a.d) & $\begin{array}{l}\text { Human: none } \\
\text { Mouse: NP_001157725.1 } \\
\text { Rat: NP_001029246.1 }\end{array}$ & $\begin{array}{l}1 \mathrm{~b} . \text { - 3.4.5.6a.7.8.9d } \\
1 \mathrm{~b} .-3.4 .5 .6 \mathrm{a} \cdot 7.8 .9 \mathrm{~d}\end{array}$ \\
\hline Tpm1.10 & $\operatorname{TmBr} 1$ & - & Tpm1.10br (a.b.b.c) & $\begin{array}{l}\text { Human: none } \\
\text { Mouse: NP_001157727.1 } \\
\text { Rat: NP_001029244.1 }\end{array}$ & $\begin{array}{l}1 \mathrm{a} \cdot 2 \mathrm{~b} \cdot 3 \cdot 4 \cdot 5 \cdot 6 \mathrm{~b} \cdot 7 \cdot 8 \cdot 9 \mathrm{c} \\
1 \mathrm{a} \cdot 2 \mathrm{~b} \cdot 3 \cdot 4 \cdot 5 \cdot 6 \mathrm{~b} \cdot 7 \cdot 8 \cdot 9 \mathrm{c}\end{array}$ \\
\hline Tpm1.11 & $\mathrm{TmBr} 2$ & - & Tpm1.11br (b.-.b.b) & $\begin{array}{l}\text { Human: none } \\
\text { Mouse: none } \\
\text { Rat: NP_062004.1 }\end{array}$ & $\begin{array}{l}1 \mathrm{~b} .=-3.4 .5 .6 \mathrm{~b} .7 .8 .9 \mathrm{~b} \\
1 \mathrm{~b}=-3.4 .5 .6 \mathrm{~b} .7 .8 .9 \mathrm{~b}\end{array}$ \\
\hline Tpm1.12 & $\mathrm{TmBr} 3$ & Tmbr $\alpha$, Tmbr $\alpha-1 \operatorname{Tm} \alpha-1.6$ & Tpm1.12br (b.-.b.c) & $\begin{array}{l}\text { Human: NP_001018008.1 } \\
\text { Mouse: NP_001157728.1 } \\
\text { Rat: NP_001288665.1 }\end{array}$ & $\begin{array}{l}1 \mathrm{~b} .-3.4 .5 .6 \mathrm{~b} .7 .8 .9 \mathrm{c} \\
1 \mathrm{~b} .-3.4 .5 .6 \mathrm{~b} .7 .8 .9 \mathrm{c}\end{array}$ \\
\hline Tpm1.13 & - & - & Tpm1.13 (b.-b.a) & $\begin{array}{l}\text { Human: none } \\
\text { Mouse: NP_001157726.1 } \\
\text { Rat: NP_001029247.1 }\end{array}$ & $\begin{array}{l}1 \mathrm{~b} .-3.4 .5 .6 \mathrm{~b} .7 .8 .9 \mathrm{a} \\
1 \mathrm{~b} . \mathbf{3} \cdot \mathbf{4} \cdot 5.6 \mathrm{~b} .7 .8 .9 \mathrm{a}\end{array}$ \\
\hline
\end{tabular}

a The accession numbers are from September, 2014 and for full-length human, mouse and rat protein sequences that have been documented at the nucleic acid level. RefSeq numbers are listed when available. The $\mathrm{N}$-terminal Met is removed post translation in all exon $1 \mathrm{~b}$-encoded sequences. As far as is currently known the N-terminal amino acid is N-acetylated post translation in all isoforms. NCBI website has been updated to include the above terminology

b The exon notation includes only the protein-coding exons and does not reflect splice variants in non-coding regions at the $3^{\prime}$ end of the mRNA transcripts 
Table 2 List of tropomyosin isoforms derived from the human TPM3 gene (geneID:7170), the mouse Tpm3 gene (geneID:59069) or the rat Tpm3 gene (geneID:117557). Gene structure: 1a

\begin{tabular}{|c|c|c|c|c|c|}
\hline $\begin{array}{l}\text { Short } \\
\text { name }\end{array}$ & $\begin{array}{l}\text { Common } \\
\text { names }\end{array}$ & $\begin{array}{l}\text { Alternate } \\
\text { names } \\
\text { in use }\end{array}$ & $\begin{array}{l}\text { Formal protein } \\
\text { name }\end{array}$ & $\begin{array}{l}\text { Accession numbers } \\
\text { Full length sequences only }\end{array}$ & Exon usage $^{\mathrm{b}}$ \\
\hline Tpm3.12 & Tm sk $\alpha$-slow & $\begin{array}{r}\gamma \operatorname{Tm} \alpha \operatorname{sm} 1 \\
\text { slow skTm }\end{array}$ & Tpm3.12st (a.b.b.a) & $\begin{array}{l}\text { Human: NP_689476.2 } \\
\text { Mouse: NP_001280677.2 Rat: } \\
\text { NP_001288214.1 }\end{array}$ & $\begin{array}{l}1 a \cdot 2 b \cdot 3 \cdot 4 \cdot 5 \cdot 6 b \cdot 7 \cdot 8 \cdot 9 a \\
1 a \cdot 2 b \cdot 3 \cdot 4 \cdot 5 \cdot 6 b \cdot 7 \cdot 8 \cdot 9 a\end{array}$ \\
\hline Tpm3.13 & - & - & Tpm3.13cy (a.b.a.d) & $\begin{array}{l}\text { Human: none } \\
\text { Mouse: NP_071709.2 } \\
\text { Rat: none }\end{array}$ & $\begin{array}{l}1 \mathrm{a} \cdot 2 \mathrm{~b} \cdot 3 \cdot 4 \cdot 5 \cdot 6 \mathrm{a} \cdot 7 \cdot 8 \cdot 9 \mathrm{~d} \\
1 \mathrm{a} \cdot 2 \mathrm{~b} \cdot 3 \cdot 4 \cdot 5 \cdot 6 \mathrm{a} \cdot 7 \cdot 8 \cdot 9 \mathrm{~d}\end{array}$ \\
\hline Tpm3.1 & Tm5NM1 & - & Tpm3.1cy(b.-.a.d) & $\begin{array}{l}\text { Human: NP_705935.1 } \\
\text { Mouse: NP_001240667.1 } \\
\text { Rat: NP_775134.1 }\end{array}$ & $\begin{array}{l}1 \mathrm{~b} \cdot \text { - 3.4.5.6a.7.8.9d } \\
1 \mathrm{~b} . \mathbf{3} \cdot \mathbf{4} \cdot 5 \cdot 6 \mathrm{a} \cdot 7.8 .9 \mathrm{~d}\end{array}$ \\
\hline Tpm3.2 & Tm5NM2 & - & $\operatorname{Tpm} 3.2 \mathrm{cy}($ b.-.b.d) & $\begin{array}{l}\text { Human: NP_001036816.1 } \\
\text { Mouse: NP_001240669.1 } \\
\text { Rat: none }\end{array}$ & 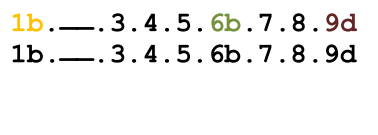 \\
\hline Tpm3.3 & Tm5NM3 & - & $\operatorname{Tpm} 3.3 c y(b .-. b . a)$ & $\begin{array}{l}\text { Human: EAW53237.1 } \\
\text { Mouse: none } \\
\text { Rat: NP_001288215.1 }\end{array}$ & $\begin{array}{l}1 \mathrm{~b} \cdot \text { - } 3 \cdot 4 \cdot 5 \cdot 6 \mathrm{~b} \cdot 7 \cdot 8 \cdot 9 \mathrm{a} \\
1 \mathrm{~b} \cdot \mathbf{3} \cdot \mathbf{4} \cdot \mathbf{5} \cdot 6 \mathrm{~b} \cdot \mathbf{7} \cdot \mathbf{9} \cdot 9 \mathrm{a}\end{array}$ \\
\hline Tpm3.4 & Tm5NM4 & - & Tpm3.4cy (b.-.b.c) & $\begin{array}{l}\text { Human: NP_001036818.1 } \\
\text { Mouse: NP_001280678.1 } \\
\text { Rat: NP_476556.2 }\end{array}$ & $\begin{array}{l}1 \mathrm{~b} .-3 \cdot 4 \cdot 5 \cdot 6 \mathrm{~b} \cdot 7.8 .9 \mathrm{c} \\
1 \mathrm{~b} .-3.4 .5 .6 \mathrm{~b} \cdot 7.8 .9 \mathrm{c}\end{array}$ \\
\hline Tpm3.5 & Tm5NM5 & - & Tpm3.5cy(b.-.a.a) & $\begin{array}{l}\text { Human: NP_001265118.1 } \\
\text { Mouse: NP_001258693.1 } \\
\text { Rat: none }\end{array}$ & $\begin{array}{l}1 \mathrm{~b} \cdot \mathbf{3} \cdot 4 \cdot 5 \cdot 6 \mathrm{a} \cdot 7 \cdot 8 \cdot 9 \mathrm{a} \\
1 \mathrm{~b} \cdot \mathbf{3} \cdot \mathbf{4} \cdot 5 \cdot 6 \mathrm{a} \cdot 7.8 .9 \mathrm{a}\end{array}$ \\
\hline Tpm3.7 & Tm5NM7 & - & Tpm3.7cy (b.-.a.c) & $\begin{array}{l}\text { Human: NP_001036817.1 } \\
\text { Mouse: none } \\
\text { Rat: none }\end{array}$ & $\begin{array}{l}1 \mathrm{~b} . \text { - 3.4.5.6a.7.8.9c } \\
1 \mathrm{~b} .-3.4 .5 \cdot 6 \mathrm{a} \cdot 7.8 .9 \mathrm{c}\end{array}$ \\
\hline Tpm3.8 & Tm5NM8 & - & Tpm3.8cy (b.-.a.a/c) ${ }^{\mathrm{c}}$ & $\begin{array}{l}\text { Human: none } \\
\text { Mouse: none } \\
\text { Rat: none }\end{array}$ & $\begin{array}{l}1 b \cdot \text { - 3.4.5.6a.7.8.9a/c } \\
1 b .-3.4 \cdot 5 \cdot 6 a \cdot 7 \cdot 8.9 a / c\end{array}$ \\
\hline Tpm3.9 & Tm5NM9 & - & Tpm3.9cy (b.-.b.a/c) ${ }^{\mathrm{c}}$ & $\begin{array}{l}\text { Human: none } \\
\text { Mouse: none } \\
\text { Rat: none }\end{array}$ & $\begin{array}{l}1 b \cdot-3 \cdot 4 \cdot 5 \cdot 6 b \cdot 7 \cdot 8 \cdot 9 \mathrm{a} / \mathrm{c} \\
1 \mathrm{~b} \cdot \mathbf{3} \cdot \mathbf{4} \cdot \mathbf{5} \cdot 6 \mathrm{~b} \cdot \mathbf{7} \cdot \mathbf{8} \cdot 9 \mathrm{a} / \mathrm{c}\end{array}$ \\
\hline
\end{tabular}

a The accession numbers are from September, 2014 and for full-length human, mouse and rat protein sequences that have been documented at the nucleic acid level. RefSeq numbers are listed when available. The N-terminal Met is removed post translation in all exon $1 \mathrm{~b}$-encoded sequences. As far as is currently known the N-terminal amino acid is N-acetylated post translation in all isoforms. NCBI website has been updated to include the above terminology

b The exon notation includes only the protein-coding exons and does not reflect splice variants in non-coding regions at the $3^{\prime}$ end of the mRNA transcripts

c The designation a/c indicates that the ninth exon includes exon 9a and five amino acids from exon $9 \mathrm{c}$

potential isoforms from the TPMI gene using RT-PCR. Our ability to detect and confirm additional isoforms will depend on both the extent of expression across, and the levels of expression in, different cell types.
The NCBI accession numbers for each isoform are included in Tables 1, 2, 3 and 4 and the human, mouse and rat genes on the NCBI website have been amended to use the nomenclature as listed here. 
Table 3 List of tropomyosin isoforms derived from the human TPM2 gene (geneID:7169), the mouse Tpm2 gene (geneID:22004) or the rat Tpm2 gene (geneID:500450) ${ }^{a}$. Gene structure $1 \mathrm{a} \quad$ 2b $1 \mathrm{~b} \quad \begin{array}{llllllll}4 & 5 & 6 a & 6 b & 7 & 8 & 9 a & 9 d\end{array}$

\begin{tabular}{|c|c|c|c|c|c|}
\hline $\begin{array}{l}\text { Short } \\
\text { name }\end{array}$ & $\begin{array}{l}\text { Common } \\
\text { names }\end{array}$ & Alternate names in use & Formal protein name & $\begin{array}{l}\text { Accession numbers } \\
\text { Full length sequences only }{ }^{\mathrm{b}}\end{array}$ & Exon usage $^{c}$ \\
\hline Tpm2.1 & $\operatorname{Tm} \operatorname{sm} \beta$ & $\begin{array}{l}\beta \operatorname{Tm} \mathrm{Tm} 1, \operatorname{Tm} 1 \beta, \text { smooth Tm, } \\
\text { smooth } \alpha-\operatorname{Tm}\end{array}$ & Tpm2.1sm/cy (a.b.a.d) & $\begin{array}{l}\text { Human: NP_998839.1 } \\
\text { Mouse: NP_001264805.1 } \\
\text { Rat: NP_001019516.1 }\end{array}$ & $\begin{array}{l}1 \mathrm{a} \cdot 2 \mathrm{~b} \cdot 3 \cdot 4 \cdot 5 \cdot 6 \mathrm{a} \cdot 7 \cdot 8 \cdot 9 \mathrm{~d} \\
1 \mathrm{a} \cdot 2 \mathrm{~b} \cdot 3 \cdot 4 \cdot 5 \cdot 6 \mathrm{a} \cdot 7 \cdot 8 \cdot 9 \mathrm{~d}\end{array}$ \\
\hline Tpm2.2 & Tm sk $\beta$ & $\beta$ skeletal Tm, $\beta$ cardiac Tm & Tpm2.2st (a.b.b.a) & $\begin{array}{l}\text { Human: NP_003280.2 } \\
\text { Mouse: NP_033442.2 } \\
\text { Rat: NP_001288164.1 }\end{array}$ & $\begin{array}{l}1 \mathrm{a} \cdot 2 \mathrm{~b} \cdot 3 \cdot 4 \cdot 5 \cdot 6 \mathrm{~b} \cdot 7 \cdot 8 \cdot 9 \mathrm{a} \\
1 \mathrm{a} \cdot 2 \mathrm{~b} \cdot 3 \cdot 4 \cdot 5 \cdot 6 \mathrm{~b} \cdot 7 \cdot 8 \cdot 9 \mathrm{a}\end{array}$ \\
\hline Tpm2.3 & & - & Tpm2.3 (a.b.b.d) & $\begin{array}{l}\text { Human: NP_001288155.1 } \\
\text { Mouse: NP_001264804.1 } \\
\text { Rat: none }\end{array}$ & $\begin{array}{l}1 \mathrm{a} \cdot 2 \mathrm{~b} \cdot 3 \cdot 4 \cdot 5 \cdot 6 \mathrm{~b} \cdot 7 \cdot 8 \cdot 9 \mathrm{~d} \\
1 \mathrm{a} \cdot 2 \mathrm{~b} \cdot 3 \cdot 4 \cdot 5 \cdot 6 \mathrm{~b} \cdot 7 \cdot 8 \cdot 9 \mathrm{~d}\end{array}$ \\
\hline Tpm2.4 & & - & $\begin{array}{l}\text { Tpm2.4 } \\
\text { (a.b.a.a) }\end{array}$ & $\begin{array}{l}\text { Human: NP_001288156.1 } \\
\text { Mouse: none } \\
\text { Rat: none }\end{array}$ & $\begin{array}{l}1 a \cdot 2 b \cdot 3 \cdot 4 \cdot 5 \cdot 6 a \cdot 7 \cdot 8 \cdot 9 a \\
1 a \cdot 2 b \cdot 3 \cdot 4 \cdot 5 \cdot 6 a \cdot 7 \cdot 8 \cdot 9 a\end{array}$ \\
\hline
\end{tabular}

a The TPM2 genes in mouse, rat and human do not contain exon 1b. It is included here as it is found in chicken and zebrafish

b The accession numbers are from September, 2014 and for full-length human, mouse and rat protein sequences that have been documented at the nucleic acid level. RefSeq numbers are listed when available. The N-terminal Met is removed post translation in all exon 1b-encoded sequences. As far as is currently known the N-terminal amino acid is N-acetylated post translation in all isoforms. NCBI website has been updated to include the above terminology

c The exon notation includes only the protein-coding exons and does not reflect splice variants in non-coding regions at the $3^{\prime}$ end of the mRNA transcripts

Table 4 List of tropomyosin isoforms derived from the human TPM4 gene (geneID:7171), the mouse Tpm4 gene (geneID:326618) or the rat gene Tpm4 (geneID:248512) ${ }^{\mathrm{a}}$. Gene structure ${ }^{1 \mathrm{a}}$

\begin{tabular}{|c|c|c|c|c|c|}
\hline Short name & Common names & $\begin{array}{l}\text { Alternate names } \\
\text { in use }\end{array}$ & Formal protein name & $\begin{array}{l}\text { Accession numbers } \\
\text { Full length sequences only }\end{array}$ & Exon usage $^{\mathrm{c}}$ \\
\hline Tpm4.1 & Tm4HMW & $\delta \mathrm{Tm}$ & Tpm4.1cy (a.b.b.d) & $\begin{array}{l}\text { Human: NP_001138632.1 } \\
\text { Mouse: none } \\
\text { Rat: none }\end{array}$ & $\begin{array}{l}1 \mathrm{a} \cdot 2 \mathrm{~b} \cdot 3 \cdot 4 \cdot 5 \cdot 6 \mathrm{~b} \cdot 7 \cdot 8 \cdot 9 \mathrm{~d} \\
1 \mathrm{a} \cdot 2 \mathrm{~b} \cdot 3 \cdot 4 \cdot 5 \cdot 6 \mathrm{~b} \cdot 7 \cdot 8 \cdot 9 \mathrm{~d}\end{array}$ \\
\hline Tpm4.2 & $\operatorname{Tm} 4$ & - & Tpm4.2cy (b.-.b.d) & $\begin{array}{l}\text { Human: NP_003281.1 } \\
\text { Mouse: NP_001001491.1 } \\
\text { Rat: NP_036810.1 }\end{array}$ & $\begin{array}{l}1 \mathrm{~b} \cdot \text { - 3.4.5.6b.7.8.9d } \\
1 \mathrm{~b} \cdot \mathbf{3} \cdot \mathbf{4} \cdot \mathbf{5} \cdot 6 \mathrm{~b} \cdot 7.8 .9 \mathrm{~d}\end{array}$ \\
\hline
\end{tabular}

a The TPM4 gene of humans (but not rat or mouse) contains exon 9a but there are no reported Tpm4 isoforms expressing exon 9a. Exon 9a is present in other vertebrates e.g., chicken, frog and zebrafish

b The accession numbers are from September, 2014 and for full-length human, mouse and rat protein sequences that have been documented at the nucleic acid level. RefSeq numbers are listed when available. The N-terminal Met is removed post translation in all exon 1b-encoded sequences. As far as is currently known the N-terminal amino acid is N-acetylated post translation in all isoforms. NCBI website has been updated to include the above terminology

c The exon notation includes only the protein-coding exons and does not reflect splice variants in non-coding regions at the $3^{\prime}$ end of the mRNA transcripts 
Table 5 Human tropomyosin amino acid sequences in order of the exons in the gene

\section{Exon 1a \\ TPM1 -MDAIKKKMQMLKLDKENALDRAEQAEADKKAAEDRSKQ \\ TPM2 -MDAIKKKMQMLKLDKENAIDRAEQAEADKKQAEDRCKQ \\ TPM3 MMEAIKKKMQMLKLDKENALDRAEQAEAEQKQAEERSKQ \\ TPM4 -MEAIKKKMQMLKLDKENAIDRAEQAEADKKAAEDKCKQ}

\section{Exon 2 a}

TPMI LEEDIAAKEKLLRVSEDERDRVLEELHKAEDSLLAAEEAAAK

TPM2 ---------------------------------

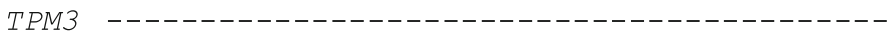

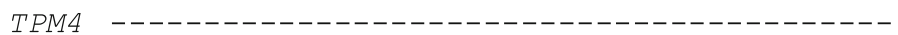

\section{Exon $2 b$}

TPM1 LEDELVSLQKKLKGTEDELDKYSEALKDAQEKLELAEKKATD

TPM2 LEEEQQALQKKLKGTEDEVEKYSESVKEAQEKLEQAEKKATD

TPM3 LEDELAAMQKKLKGTEDELDKYSEALKDAQEKLELAEKKAAD

TPM4 VEEELTHLQKKLKGTEDELDKYSEDLKDAQEKLELTEKKASD

\section{Exon 1b}

TPMI MAGSSSLEAVRRKIRSLQEQADAAEERAGTLQRELDHERKLRET

TPM2 -------------------------------------------

TPM3 MAGITTIEAVKRKIQVLQQQADDAEERAERLQREVEGERRAREQ

TPM4 MAGLNSLEAVKRKIQALQQQADEAEDRAQGLQRELDGERERREK

Exon 3

TPMI AEADVASLNRRIQLVEEELDRAQERLATALQKLEEAEKAADESER

TPM2 AEADVASLNRRIQLVEEELDRAQERLATALQKLEEAEKAADESER

TPM3 AEAEVASLNRRIQLVEEELDRAQERLATALQKLEEAEKAADESER

TPM4 AEGDVAALNRRIQLVEEELDRAQERLATALQKLEEAEKAADESER

\section{Exon 4}

TPM1 GMKVIESRAQKDEEKMEIQEIQLKEAKHIAEDADRKYEE

TPM2 GMKVIENRAMKDEEKMELQEMQLKEAKHIAEDSDRKYEE

TPM3 GMKVIENRALKDEEKMELQEIQLKEAKHIAEEADRKYEE

TPM4 GMKVIENRAMKDEEKMEIQEMQLKEAKHIAEEADRKYEE

Exon 5

TPMI VARKLVIIESDLERAEERAELSEG

TPM2 VARKLVILEGELERSEERAEVAES

TPM3 VARKLVIIEGDLERTEERAELAES

TPM4 VARKLVILEGELERAEERAEVSEL

\section{Exon $6 a$}

TPM1 QVRQLEEQLRIMDQTLKALMAAEDK

TPM2 RARQLEEELRTMDQALKSLMASEEE

TPM3 RCREMDEQIRLMDQNLKCLSAAEES

TPM4 -----------------------

\section{Exon 7}

TPM1 YSQKEDRYEEEIKVLSDKLKE

TPM2 YSTKEDKYEEEIKLLEEKLKE

TPM3 YSQKEDKYEEEIKILTDKLKE

TPM4 YSEKEDKYEEEIKLLSDKLKE

\section{Exon $6 b$}

KCAELEEELKTVTNNLKS LEAQAEK KCGDLEEELKIVTNNLKSLEAQADK KCSELEEELKNVTNNLKSLEAQAEK KCGDLEEELKNVTNNLKSLEAASEK

\section{Exon 8}

AETRAEFAERSVTKLEKS IDDLE AETRAEFAERSVAKLEKTIDDLE AETRAEFAERSVAKLEKTIDDLE AETRAEFAERTVAKLEKTIDDLE 
Table 5 continued

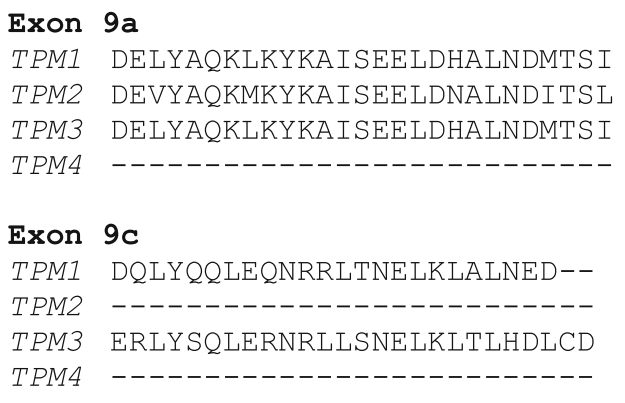

Exon 9b

DKELCFTSPKTPSSSWMSHLSELCICLFSS

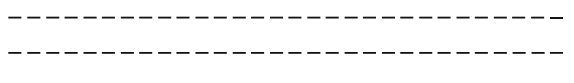

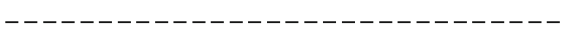

\section{Exon 9d}

EKVAHAKEENLSMHQMLDQTLLELNNM

ETLASAKEENVEIHQTLDQTLLELNNL

DKLKCTKEEHLCTQRMLDQTLLDLNEM

EKLAQAKEENVGLHQTLDQTLNELNCI

The exon sequences are in the order of the coding exons in the genes. Accession numbers used to compile the sequences:

$\begin{array}{ll}\text { TPM1 } & \text { NM_001018020.1 } \\ & \text { NM_001018006.1 } \\ & \text { NM_000366.5 } \\ & \text { NM_001018005.1 } \\ & \text { NM_001018008.1 } \\ & \text { NM_001018004.1 } \\ & \text { NM_001018007.1 } \\ & \text { EL947365, DB542398 } \\ & \text { NM_213674.1 } \\ \text { TPM2 } & \text { NM_003289.3 } \\ & \text { NM_152263.2 } \\ \text { TPM3 } & \text { NM_153649.3 } \\ & \text { NM_001043352.1 } \\ & \text { NM_001043351.1 } \\ & \text { NM_001043353.1 } \\ & \text { NM_003290.2 } \\ \text { TPM4 } & \text { NM 001145160.1 }\end{array}$

For additional information about tropomyosin sequences and exon organization in animals, refer to Barua et al. 2011 and Schevzov et al. 2011

Acknowledgments This work was supported by the following grants National Institutes of Health (USA): GM-093065 to SEH-D and Wellcome Trust Programme Grant 085309 to MAG. We thank Michael Murphy and Lillian Riddick at the NCBI for their assistance in verifying accession numbers and for including the proposed nomenclature in the NCBI website.

Open Access This article is distributed under the terms of the Creative Commons Attribution License which permits any use, distribution, and reproduction in any medium, provided the original author(s) and the source are credited.

\section{References}

Bailey K (1946) Tropomyosin: a new asymmetric protein component of muscle. Nature 157:368

Barua B, Pamula MC et al (2011) Evolutionarily conserved surface residues constitute actin binding sites of tropomyosin. Proc Nat Acad Sci USA 108(25):10150-10155

Cooley BC, Bergtrom G (2001) Multiple combinations of alternatively spliced exons in rat tropomyosin-alpha gene mRNA: evidence for 20 new isoforms in adult tissues and cultured cells. Arch Biochem Biophys 390(1):71-77

Cranz-Mileva S, Pamula MC et al (2013) A molecular evolution approach to study the roles of tropomyosin in fission yeast. PLoS ONE 8(10):e76726

Geeves MA (2012) Thin filament regulation. Compr Biophys 4:251-267

Gordon AM, Homsher E et al (2000) Regulation of contraction in striated muscle. Physiol Rev 80(2):853-924

Gunning P, O'Neill G et al (2008) Tropomyosin-based regulation of the actin cytoskeleton in time and space. Physiol Rev 88(1):1-35

Marston SB, Gautel M (eds) (2013) Tropomyosin form and function. J Muscle Res Cell Motil 34(3-4):1573-2657

Pittenger MF, Kazzaz JA et al (1994) Functional properties of nonmuscle tropomyosin isoforms. Curr Opin Cell Biol 6(1):96-104

Schevzov G, Whittaker SP et al (2011) Tropomyosin isoforms and reagents. Bioarchitecture 1(4):135-164

Vindin H, Gunning P (2013) Cytoskeletal tropomyosins: choreographers of actin filament functional diversity. J Muscle Res Cell Motil 34(3-4):261-274 